\title{
Lack of synergistic interaction between quercetin and catechin in systemic and pulmonary vascular smooth muscle
}

\author{
Carmen Menendez ${ }^{1,2}$, Rosario Jimenez ${ }^{3}$, Laura Moreno ${ }^{1,2}$, Pilar Galindo ${ }^{3}$, Angel Cogolludo ${ }^{1,2}$, \\ Juan Duarte ${ }^{3}$ and Francisco Perez-Vizcaino ${ }^{1,2_{*}}$ \\ ${ }^{1}$ Department of Pharmacology, School of Medicine, University Complutense of Madrid, 28040 Madrid, Spain \\ ${ }^{2}$ Ciber Enfermedades Respiratorias (CIBERES), 28040 Madrid, Spain \\ ${ }^{3}$ Department of Pharmacology, School of Pharmacy, University of Granada, 18071 Granada, Spain
}

(Received 28 April 2010 - Revised 5 October 2010 - Accepted 1 November 2010 - First published online 10 December 2010)

\section{Abstract}

Due to their ubiquitous distribution, flavonoids from different classes are commonly present together in foods. However, little is known about the interactions between them. The flavonol quercetin and the flavan-3-ol $(+)$-catechin are among the most abundant flavonoids in the diet. In the present study, we have analysed the interactions between these two flavonoids on vascular function using two pure compounds and mixtures of these flavonoids in 1:0.1, 1:1 or 1:10 proportions. Quercetin induced a more potent concentration-dependent relaxant effect than catechin in the isolated rat aorta, and the isobolographic analysis of the mixtures showed no synergistic or antagonistic effects between them, i.e. their effects were additive. Quercetin was more potent in mesenteric than in pulmonary arteries. Catechin had weak effects in these vessels and did not modify the effects of quercetin. Endothelial dysfunction induced by increased oxidative stress by the superoxide dismutase inhibitor diethyldithiocarbamate was prevented by quercetin, whereas catechin showed a weak effect and the $1: 1$ mixture an intermediate effect compared with the pure compounds. Quercetin but not catechin showed a pro-oxidant and NO-scavenging effect, which was not prevented by catechin. In conclusion, catechin was less potent than quercetin as a vasodilator, pro-oxidant or to prevent endothelial dysfunction, and there were no synergistic interactions between quercetin and catechin.

\section{Key words: Quercetin: Catechin: Isobologram: Endothelium}

Flavonoids are polyphenolic compounds ubiquitously distributed in plants and are consumed regularly in the diet in considerable amounts in the form of fruits, vegetables, nuts and derived products such as red wine, tea and chocolate. Based on their chemical structure, flavonoids can be classified into several subclasses such as flavonols, flavones, flavanones, flavan-3-ols (also referred to as catechins), anthocyanidins, isoflavones, dihydroflavonols and chalcones. Flavan-3-ols and flavonols are the most abundant and are widely distributed in food. The average daily intake in the occidental diet of flavonols is estimated to be approximately $20-25 \mathrm{mg}$, with quercetin contributing $60-75 \%$ of the total ${ }^{(1,2)}$. The average intake of flavan-3-ols is within a similar range (approximately $18-31 \mathrm{mg}$ ), with (+)-catechin (henceforth referred to as catechin) and epicatechin being the most abundant ${ }^{(3)}$.

Prospective epidemiological studies have shown an inverse correlation between dietary flavonoid intake and mortality from $\mathrm{CHD}^{(1,2)}$. Several studies using various animal models have provided support for the observed protective effects of dietary flavonoids with respect to $\mathrm{CVD}^{(4-6)}$. However, the profiles of biological effects show marked differences among the different classes of flavonoids ${ }^{(7)}$. For example, flavonols exert systemic and coronary vasodilatation ${ }^{(8,9)}$, while catechins exhibit a weak vasodilator effect ${ }^{(8)}$. Both flavonoid groups show antioxidant effects in vitro and can prevent endothelial dysfunction in vivo ${ }^{(10,11)}$

Due to their ubiquitous distribution, flavonoids from different classes are commonly present together in foods and/or are consumed in the same meal. In fact, prototypical flavonoidrich foods contain a large number of different flavonoids in variable amounts. However, little is known about the interactions between them. Quercetin and catechin have been reported to act synergistically in reducing platelet recruitment via the inhibition of protein kinase C-dependent NADPH

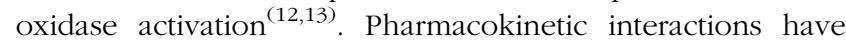
also been described ${ }^{(14)}$

In the present study, we have analysed the interactions between quercetin and catechin, the representative compounds of the two major classes of flavonoids, on vascular function in vitro.

Abbreviations: DETCA, diethyldithiocarbamate; DHE, dihydroethidium

*Corresponding author: F. Perez-Vizcaino, email fperez@med.ucm.es 


\section{Experimental methods}

The present study was conducted according to the Guide for the Care and Use of Laboratory Animals published by the US National Institutes of Health (NIH Publication no. 85-23, revised 1996) and approved by our Institutional Committee (Comité de de Experimentación animal, Universidad Complutense) for the ethical care of animals. Male Wistar rats were obtained from Harlan Laboratories.

\section{Materials and solutions}

All drugs and chemicals were purchased from Sigma (St Louis, MO, USA). Flavonoids were dissolved in dimethyl sulphoxide, and all the other compounds were dissolved in distilled, deionised water. Quercetin-catechin mixtures were prepared by mixing the stock solutions, and the concentrations of mixtures are expressed as the sum of both, e.g. a $10 \mu \mathrm{m}$-quercetincatechin (1:10) mixture contains 0.91 $\mathrm{m}$-quercetin and 9.09 $\mu \mathrm{m}$-catechin. To prepare the NO solutions used for biological activity, a vial containing $20 \mathrm{ml}$ of Krebs solution at $37^{\circ} \mathrm{C}$ was initially bubbled with $\mathrm{N}_{2}$ for $15 \mathrm{~min}$ and then continuously bubbled with $\mathrm{NO}$ (450 parts per million from Air Liquide España, Paris, France), resulting in a concentration of 0.9-1 $\mu \mathrm{M}$ (as measured by an ISO-NO meter electrochemical electrode; WPI, Stevenage, Herts, UK). The Krebs solution contained $118 \mathrm{~mm}-\mathrm{NaCl}, 4.75 \mathrm{~mm}-\mathrm{KCl}, 25 \mathrm{~mm}-\mathrm{NaHCO}_{3}, 1.2 \mathrm{~mm}-$ $\mathrm{MgSO}_{4}, 2 \mathrm{~mm}-\mathrm{CaCl}_{2}, 1.2 \mathrm{~mm}-\mathrm{KH}_{2} \mathrm{PO}_{4}$ and $11 \mathrm{~mm}$-glucose. The HEPES-buffered solution contained $130 \mathrm{~mm}-\mathrm{NaCl}, 5 \mathrm{~mm}-\mathrm{KCl}$,

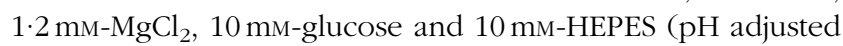
to $7 \cdot 4$ with $\mathrm{NaOH})$.

\section{Auto-oxidation}

Absorption spectra of quercetin and catechin in the Krebs solution, bubbled with $95 \% \mathrm{O}_{2}$ and $5 \% \mathrm{CO}_{2}$ at $37^{\circ} \mathrm{C}, \mathrm{pH} 7 \cdot 4$, were analysed in a spectrophotometer (6405; Jenway, BioWorld, Bibby Scientific Ltd, Staffs, UK) ${ }^{(15)}$. Spectra were obtained just after dilution and at 10, 20, 60 and $120 \mathrm{~min}$.

\section{Nitric oxide scavenging}

NO was monitored with the ISO-NO meter in a $20 \mathrm{ml}$ chamber at $37^{\circ} \mathrm{C}$ filled with the HEPES-buffered solution as described previously ${ }^{(15)}$. At the beginning of the experiment, the system was closed with no headspace; under constant rapid stirring, NO concentration was approximately $100 \mathrm{~nm}$ and $\mathrm{O}_{2}$ concentration was $20 \%$, and the $\mathrm{pH}$ was $7 \cdot 4$.

\section{Aortic contractile tension recording}

Thoracic aortic rings $(3 \mathrm{~mm}$ in length) from Wistar rats were mounted in organ chambers for isometric force recording ${ }^{(16)}$. In some experiments, aortae were mechanically denuded of endothelium by rubbing the luminal surface with a metal rod. Aortic rings were stretched to $2 \mathrm{~g}$ of tension and equilibrated for $90-120 \mathrm{~min}$. During this period, Krebs solution was changed every $30 \mathrm{~min}$, and the rings were re-stretched as needed to maintain a final tension of $2 \mathrm{~g}$. Endothelium denudation was confirmed by the absence of a relaxant response to acetylcholine $(<10 \%)$. To analyse the effects on endothelial function and its inhibition by the superoxide dismutase inhibitor diethyldithiocarbamate (DETCA), endothelium-intact rings were pre-incubated in the absence or presence of DETCA (1 mM) and with vehicle (dimethyl sulphoxide $0 \cdot 1 \%$ ), quercetin, catechin or the quercetin-catechin mixture $(1: 1 ; 10 \mu \mathrm{M})$ for $10 \mathrm{~min}$. After stimulation with phenylephrine (100 nm), a concentration-response curve analysis to acetylcholine was performed.

\section{Contraction in mesenteric and pulmonary resistance arteries}

Mesenteric and pulmonary arterial rings (internal diameter $300-500 \mu \mathrm{m}$ ) were mounted in a wire myograph in Krebs solution and stretched to give an equivalent transmural pressure of $100 \mathrm{mmHg}^{(17)}$. After equilibration, arteries were exposed to U46619 $(0 \cdot 1 \mu \mathrm{M})$, and after a steady-state constrictor response was obtained, the flavonoids quercetin, catechin or their mixtures were added cumulatively. The NO synthase inhibitor G-nitro-L-arginine-methyl ester ( $0.1 \mathrm{~mm})$ was added at the end of the experiment to test the reversal of flavonoid-induced relaxation.

\section{Pro-oxidant effect in rat aortic smooth muscle cells in culture}

Primary cultures of smooth muscle cells were prepared from rat aortic explants as described previously ${ }^{(18)}$. Dihydroethidium (DHE) enters the cells and is oxidised by superoxide to yield ethidium, which binds to DNA to produce bright red fluorescence. Cells cultured in ninety-six-well plates (passages 2-3) were incubated in HEPES-buffered solution containing DHE $(10 \mu \mathrm{M})$ at $37^{\circ} \mathrm{C}$ for $30 \mathrm{~min}$, and then exposed for another $30 \mathrm{~min}$ to various concentrations of quercetin, catechin or their mixtures. DHE fluorescence was read in a fluoroskan fluorometer (Thermoscientific, Madison, WI, USA). Excitation and emission were filtered at 530 and $620 \mathrm{~nm}$, respectively).

\section{Statistical analysis}

Results are expressed as means with their standard errors, and $n$ describes the number of measurements made from different animals. Differences between experimental groups were analysed using the unpaired Student's $t$ test or, for multiple comparisons, using one-way ANOVA followed by Dunnett's post hoc test. $P$ values $<0.05$ were considered statistically significant. Individual cumulative concentration-response curves were analysed, and $\mathrm{IC}_{30}$, i.e. the concentration producing a relaxation of $30 \%$ of the previous tone, was calculated. For the isobolographic analysis, the calculated $\mathrm{IC}_{30}$ value of quercetin or catechin reflected the estimated concentration present in the mixture when a relaxation of $30 \%$ was achieved. 


\section{Results}

\section{Vasodilator effects in the rat aorta}

As reported previously, quercetin-induced a concentrationdependent relaxant effect (Fig. 1(a)). Catechin was less potent, and the $50 \%$ relaxation was not achieved at the maximal concentration $(100 \mu \mathrm{M})$ tested. The 1:0 1 quercetin-catechin mixture (90.9\% quercetin and $9 \cdot 1 \%$ catechin) produced an effect similar to quercetin, and the 1:10 mixture (9.1\% quercetin and $90.9 \%$ catechin) produced a similar effect to catechin. To analyse the interactions between the two compounds, we performed an isobolographic analysis ${ }^{(19,20)}$ from the data shown in Fig. 1(a) by plotting the $\mathrm{IC}_{30}$ values of the
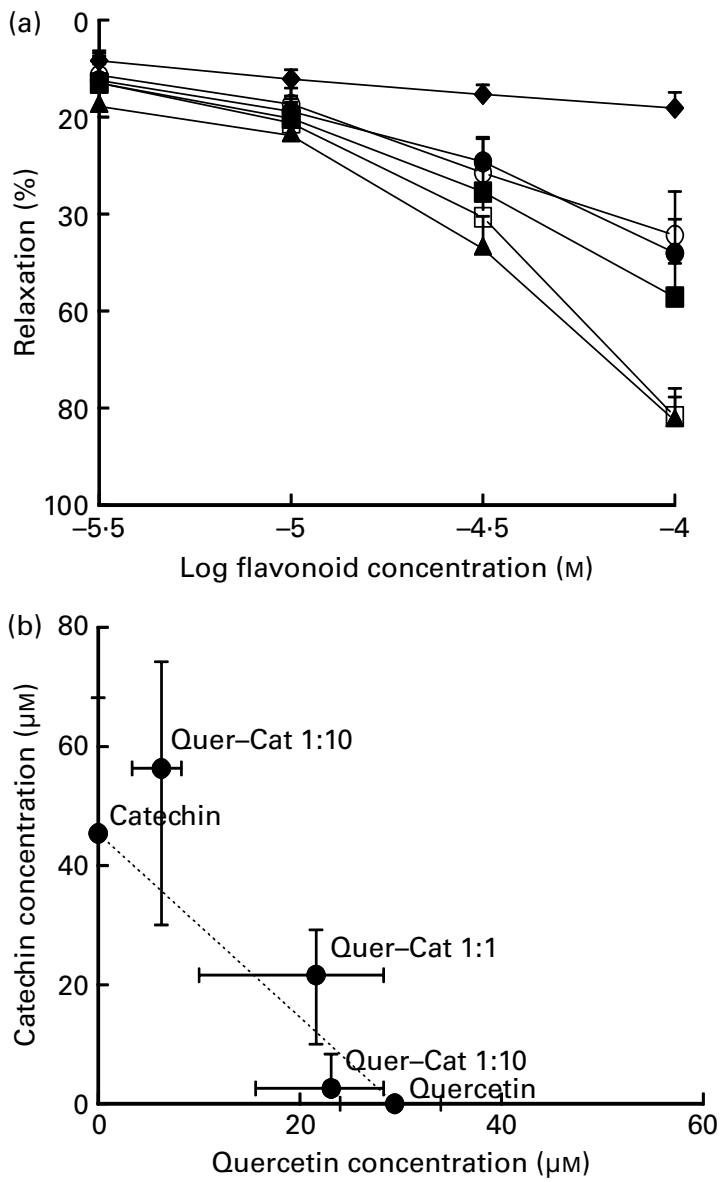

Fig. 1. Quercetin (Quer) and catechin (Cat) induce additive vasodilatory effects. Vasorelaxant responses induced by quercetin, catechin and the $1: 0 \cdot 1,1: 1$ and $1: 10$ quercetin-catechin mixtures in the endothelium-denuded rat aorta stimulated with noradrenaline $(1 \mu \mathrm{M})$. (a) Cumulative concentrationresponse curves. The concentrations of the mixtures are expressed as the sum of both compounds, e.g. in the 1:0.1 quercetin-catechin mixture at $10 \mu \mathrm{M}$ ( $\log [q u e r c e t i n 1-0 \cdot 1]=-5)$, the concentrations of each compound are $9.09 \mu \mathrm{M}$ for quercetin and $0.91 \mu \mathrm{M}$ for catechin. $O$, Catechin; $\square$, quercetin;

$\Delta$, Quer-Cat 1:0.1;, Quer-Cat 1:1; ๑, Quer-Cat 1:10; $\bullet$, DMSO. (b) Isobologram for the interaction between quercetin and catechin in causing relaxation in endothelium-denuded aortic rings. Each point represents the estimated concentration of the drug present in the mixture when a $30 \%$ relaxation is achieved, calculated from the data in (a). Results are means from six to eight experiments (except dimethyl sulphoxide (DMSO), where $n 3$ ) with standard errors represented by vertical bars. The dotted line represents the line of additivity; deviations above and below the line would show negative and positive interactions, respectively. pure compounds and their mixtures (Fig. 1(b)). The dotted line in this figure represents the line of additivity, so that deviations above and below this line indicate negative and positive interactions, respectively. It can be observed that the points of the quercetin-catechin mixtures were not significantly different from the line of additivity, indicating that there were no interactions between the two flavonoids, i.e. their effects were additive.

\section{Vasodilator effects in mesenteric and pulmonary resistance arteries}

In endothelium-intact small arteries mounted in a myograph, the contractile responses were not as sustained as in the aortae, i.e. after 70-90 min, the tone usually declined by more than $30 \%$. Therefore, only three concentrations of the flavonoids (1, 10 and $100 \mu \mathrm{M})$ could be tested. As expected ${ }^{(21)}$, quercetin produced a stronger relaxant response in the small arteries than in the aorta that was also significantly more potent in systemic (mesenteric) than in pulmonary arteries, with $10 \mu \mathrm{m}$ producing approximately 90 and $50 \%$ relaxation, respectively (Fig. 2). Addition of G-nitro-L-arginine-methyl ester at the end of the experiment could not reverse the vasodilator effects of quercetin. In contrast, $100 \mu \mathrm{M}$-catechin produced only approximately $20 \%$ relaxation in both arteries. Despite the use of only three concentrations and the weak effect of catechin precluded the isobolographic analysis of the interactions, the results obtained suggest the lack of the interaction between the two flavonoids, e.g. the 1:0 1 quercetin-catechin mixture produced effects similar to those of quercetin, and the effects of the 1:1 mixture were weakly less pronounced than those of quercetin in pulmonary arteries.

\section{Effects on endothelial function and dysfunction}

In endothelium-intact aortic rings pre-contracted with phenylephrine, acetylcholine induced a relaxant response due to the endothelial release of NO. In the presence of quercetin, catechin or the 1:1 quercetin-catechin mixture at $10 \mu \mathrm{m}$, this relaxant response was unaffected (Fig. 3(a)). The $\mathrm{Cu}^{2+}$ chelator DETCA ( $1 \mathrm{~mm}$ ) increases superoxide by inhibiting endogenous $\mathrm{Cu} / \mathrm{Zn}$ superoxide dismutase activity and hence induces NO inactivation. As expected, DETCA induced a marked impairment of endothelial-derived NO response as indicated by the reduction in the relaxant response to acetylcholine (Fig. 3(b)). Under these conditions, $10 \mu \mathrm{m}$-quercetin increased acetylcholine-induced relaxation, i.e. it prevented oxidative stress-induced endothelial dysfunction. Catechin produced a weak, borderline significant $(P=0.06)$ increase, and the $1: 1$ quercetin-catechin mixture produced an intermediate effect between the pure compounds.

\section{Pro-oxidant and nitric oxide-scavenging effects in cell-free} systems

Quercetin is oxidised in aqueous buffers, generates superoxide and inactivates $\mathrm{NO}^{(15)}$. Its oxidation can be monitored by the changes in UV spectra, i.e. an increase in absorbance 


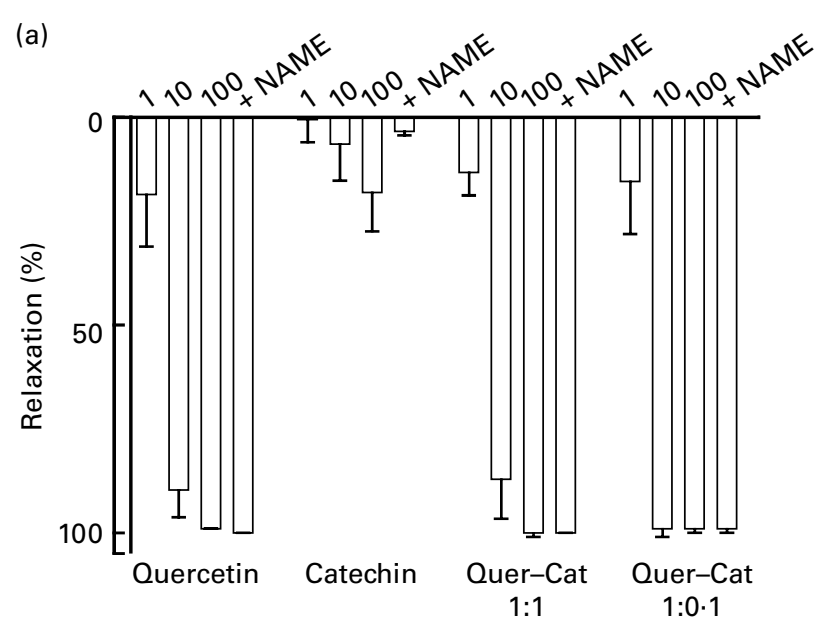

(b)

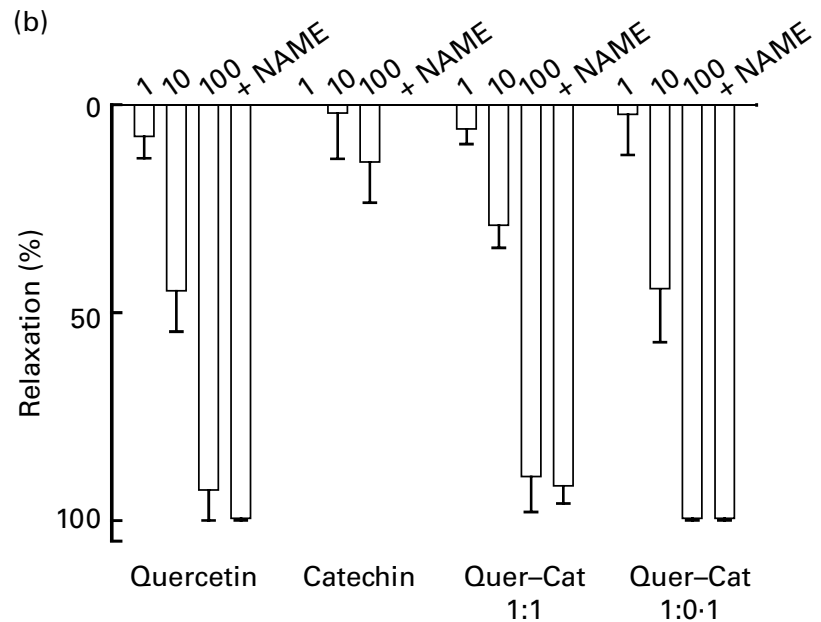

Fig. 2. Vasorelaxant responses induced by quercetin (Quer), catechin (Cat) and the 1:0.1 and 1:1 quercetin-catechin mixtures in (a) mesenteric and (b) pulmonary resistance arteries stimulated with U46619 (100 nM). After the last concentration of the flavonoids, the nitric oxide synthase inhibitor G-nitroL-arginine-methyl ester (L-NAME; $0.1 \mathrm{mM}$ ) was added to test the possible reversion of flavonoid-induced vasodilation. Results are means from five to eight experiments, with standard errors represented by vertical bars.

at $320 \mathrm{~nm}$ and a decrease at $385 \mathrm{~nm}$. Catechin $(10 \mu \mathrm{m})$, which has negligible absorbance in this wavelength range, was not able to prevent the oxidation of quercetin in the media (data not shown). Fig. 4(a) shows the inactivation of NO induced by quercetin. In contrast, the rate of decay of NO in the presence of catechin was similar to that observed in the presence of the vehicle dimethyl sulphoxide. Moreover, catechin did not alter the NO-scavenging effect induced by quercetin (Fig. 4(b)).

\section{Pro-oxidant effect in cultured aortic smooth muscle cells}

In the absence of DHE, neither $30 \mu \mathrm{m}$-quercetin nor $100 \mu \mathrm{M}$ catechin induced any change in red fluorescence. Addition of quercetin to aortic smooth muscle cells produced a concentration-dependent increase in DHE red fluorescence, indicating an increase in intracellular superoxide (Fig. 5). In contrast, catechin produced a negligible effect (not significant compared with dimethyl sulphoxide) at concentrations up to $100 \mu \mathrm{m}$. The 1:10 quercetin-catechin mixture produced a concentration-dependent increase in DHE fluorescence. This latter effect was equivalent to that produced by one-tenth of pure quercetin, e.g. $100 \mu \mathrm{m}$ of the mixture containing $9 \cdot 1 \mu \mathrm{M}$ of quercetin produced an effect similar to $10 \mu \mathrm{m}$ of pure quercetin, indicating that catechin does not interfere with the pro-oxidant effects of quercetin in smooth muscle cells.

\section{Discussion}

Endothelial, smooth muscle cells and platelets are three main targets for the actions of flavonoids regarding CVD. A synergistic interaction between catechin and quercetin to inhibit platelet aggregation has been described ${ }^{(12,13)}$. In the present study, we show that compared with quercetin, catechin produced weak vasodilator effects in conductance and resistance arteries, a weak ability to modulate oxidative stress-induced endothelial dysfunction and did not show pro-oxidant effects. Importantly, catechin did not influence the effects of quercetin on these parameters.
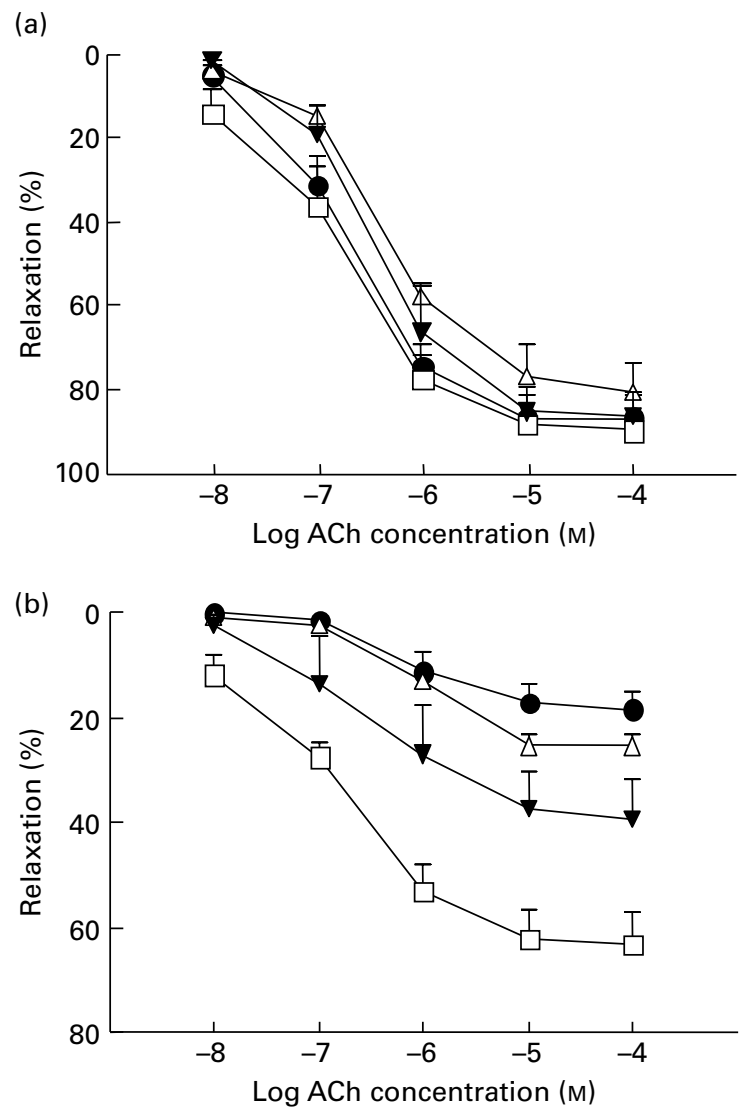

Fig. 3. (a) Effects of quercetin, catechin and the 1:1 quercetin-catechin (Quer-Cat) mixture on the endothelium-dependent vasorelaxation of acetylcholine (ACh) under control conditions and (b) after inducing endothelial dysfunction with the superoxide dismutase inhibitor diethyldithiocarbamate. Endothelium-intact aortic rings were treated for $10 \mathrm{~min}$ with the flavonoids $(10 \mu \mathrm{M})$, then stimulated with phenylephrine $(100 \mathrm{nM})$, and a concentrationresponse curve to Ach $(0.01-100 \mu \mathrm{M})$ was carried out in a cumulative fashion. Results are means of five to eight experiments, with standard errors represented by vertical bars. @, Dimethyl sulphoxide (DMSO); $\square$, quercetin; $\triangle$, catechin; $\nabla$, Quer-Cat 1:1. 

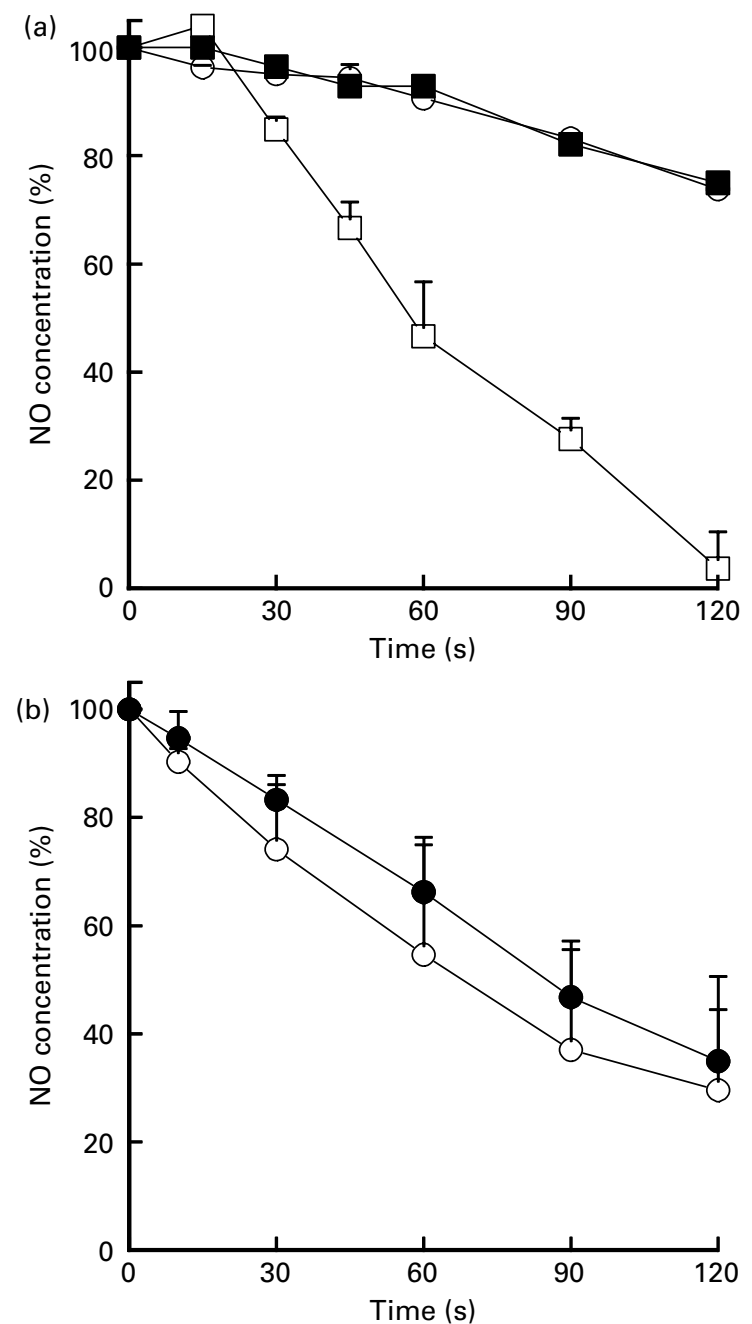

Fig. 4. Catechin does not scavenge nitric oxide and does not prevent quercetin-induced nitric oxide scavenging. (a) Time-course of nitric oxide decay in the presence of quercetin $(\square)$, catechin $(O)$ and $0.1 \%$ dimethyl sulphoxide (DMSO ( $\square$ ); vehicle). (b) Nitric oxide decay induced by quercetin in the presence of DMSO (vehicle) (๑) and catechin (O). Results are means of three experiments, with standard errors represented by vertical bars.

A direct endothelium-independent vasodilator effect of flavonols is one of the potential mechanisms involved in their anti-hypertensive effects, which might also contribute to prevent other forms of CVD such as IHD and stroke. Interestingly, quercetin is a more potent vasodilator in smaller arteries, which is involved in the control of vascular resistance and therefore regulates tissue flow and blood pressure ${ }^{(21)}$. This difference may reflect the diverse signalling involved in the contraction in large and small arteries. Recently, pulmonary arterial pressure has been implicated as a cardiovascular risk factor ${ }^{(22)}$. Herein, we report for the first time that quercetin also relaxes pulmonary resistance arteries. However, the vasodilator potency of quercetin was higher in pulmonary than in the aorta but yet lower than in systemic (mesenteric) resistance arteries, indicating a low selectivity for the pulmonary circulation. In contrast, catechin had a weak vasodilator effect. Despite some structural similarities between catechin and quercetin, the latter is planar but the former is not, which may explain some of the differences found in the biological activity. Moreover, catechin did not modify the vasodilator effects of quercetin in the three vessels studied. In the aorta, the isobolographic analysis revealed that the effects of both flavonoids were additive.

The mechanisms of drug interactions (synergism, additivity or antagonism) are most often analysed intuitively, without a proper mathematical method. The isobolographic analysis is an easy and appropriate method for the study of drug-drug interactions when both drugs show activity ${ }^{(20)}$. In the aorta, the isobologram revealed that the vasodilator effects of both flavonoids were additive because the points for their combinations were near the line of additivity (within the standard errors of the points). In mesenteric and pulmonary arteries, the isobologram could not be plotted because of the weak effect of catechin. However, the data also suggest a lack of interaction.

Endothelial dysfunction is an early and independent predictor of poor prognosis in most forms of CVD. One of the key mechanisms of endothelial dysfunction involves the vascular production of reactive oxygen species, particularly superoxide derived from NADPH oxidase, which reacts rapidly with NO and induces NO inactivation. Quercetin restores the impaired endothelial function in vitro ${ }^{(23)}$ and in vivo in several animal models of hypertension ${ }^{(24)}$ by at least two mechanisms. Acutely, quercetin directly scavenges superoxide and inhibits the activity of superoxide-generating enzymes. In the chronic phase, quercetin can prevent the expression of genes involved in superoxide generation such as the NADPH oxidase subunits induced by pathological stimuli such as angiotensin II or endothelin-1. Herein, we used a protocol to induce acute endothelial dysfunction based purely on oxidative stress by means of inhibiting the degradation of endogenous superoxide. Compounds scavenging intracellular superoxide or inhibiting the synthesis are expected to protect this (and possibly other) form of endothelial dysfunction. Catechin at

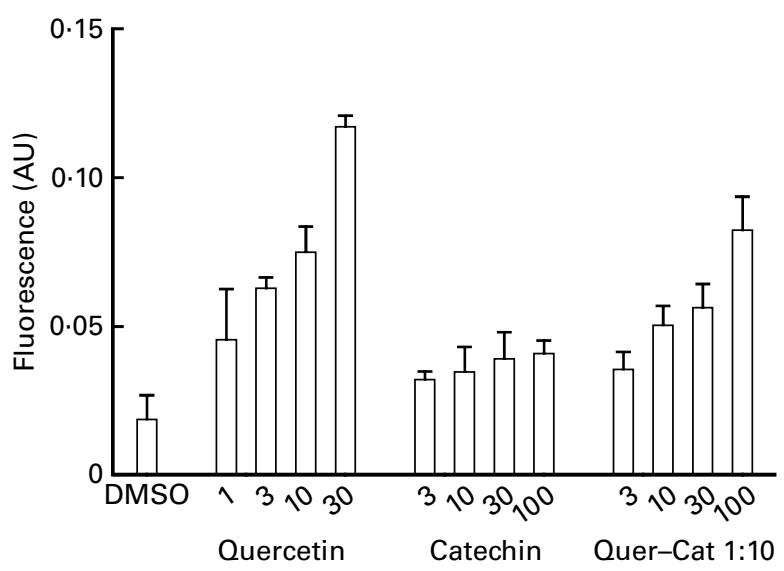

Fig. 5. Intracellular superoxide generation in aortic smooth muscle cells in primary culture. Cells incubated with the red fluorescent dye dihydroethidium were exposed to different concentrations of quercetin, catechin and the 1:10 quercetin-catechin (Quer-Cat) mixture. Fluorescence was measured at $30 \mathrm{~min}$. Results are means of two to three experiments performed in triplicate, with standard errors represented by vertical bars. AU, arbitrary units; DMSO, dimethyl sulphoxide. 
physiologically relevant concentrations of $10 \mu \mathrm{m}$ had a weak protective effect on the impairment of acetylcholine-induced relaxation by DETCA. The combination of catechin and quercetin both at $5 \mu \mathrm{m}$ produced less effective protection than $10 \mu \mathrm{m}$-quercetin, ruling out a synergistic interaction between the two compounds.

Antioxidants often also show pro-oxidant effects when analysed under specific experimental conditions. In fact, besides the antioxidant effect, flavonoids also show pro-oxidant effects $^{(15,25)}$, which may be involved in some of their biological properties. Under certain experimental conditions, the generation of superoxide by quercetin can scavenge $\mathrm{NO}$ and hence reduce NO-induced vasodilation ${ }^{(15)}$. In addition, the activation of large conductance potassium channels by quercetin involves the generation of $\mathrm{H}_{2} \mathrm{O}_{2}^{(26)}$, and this effect contributes at least partially to the coronary vasodilator effect. Quercetin is auto-oxidised and generates superoxide that rapidly reacts with $\mathrm{NO}^{(15)}$. In contrast, catechin at $10 \mu \mathrm{M}$ lacked NO-scavenging effects. The effects of quercetin on NO can be prevented by superoxide scavengers such as superoxide dismutase. It could be expected that catechin could prevent the effects of quercetin via an antioxidant effect. However, at least at this concentration of $10 \mu \mathrm{m}$, catechin did not prevent quercetin auto-oxidation nor the NOscavenging effect. Intracellular reactive oxygen species can be detected by using fluorescent dyes such as DHE, which detects superoxide, or dichlorofluorescein, which detects mainly $\mathrm{H}_{2} \mathrm{O}_{2}$. Confirming our previous report using dichlorofluorescein in coronary arteries ${ }^{(26)}$, in the present study, we also found that quercetin increases intracellular reactive oxygen species in aortic smooth muscle. Consistent with a weak NO-scavenging effect in cell-free systems, catechin did not significantly increase intracellular superoxide. Synergistic effects of polyphenolic mixtures (including catechin, quercetin, hesperidin and ferulic acid) in preventing human LDL oxidation have been found ${ }^{(27)}$. However, catechin alone did not prevent the pro-oxidant effect of quercetin either in cell-free systems or in cultured smooth muscle cells.

A limitation of the present study is that the concentration range of plasma quercetin levels in human subjects consuming flavonoid-rich foods is $0 \cdot 3-7 \cdot 6 \mu \mathrm{M}^{(28)}$, which is below the range of effective concentrations used herein $(1 \mu \mathrm{m}$ had almost no effect and $10 \mu \mathrm{m}$ had almost full inhibitory effect in the mesentery). Moreover, quercetin is mostly present in the plasma in the form of glucuronidated and sulphated metabolites ${ }^{(28)}$, which lack a direct acute vasorelaxant effect $^{(29)}$. Recent evidence has suggested that glucuronides deconjugate and the free quercetin (aglycone) is accumulated in cells at variable concentrations ${ }^{(30)}$, but its biological relevance is yet unknown.

In conclusion, the present in vitro study shows that catechin has weak effects on vascular function acutely and, when present, its effects are additive with those of quercetin, excluding synergistic interactions of these two flavonoids at this level.

\section{Acknowledgements}

The present study was supported by grants from the Ministerio de Ciencia e Innovación (AGL2007-06685, SAF2008-03948, Red HERACLES RD06/0009 and C. Menendez FPI studentship), Junta de Andalucía (P06-CTS-01555 and R. Jimenez 'Retorno de Doctores' contract) and Mutua Madrileña. The authors are grateful to Enrique Moreno and Bianca Barreira for their excellent technical assistance. The authors declare no conflicts of interest. F. P.-V. and J. D. designed the experiments, F. P.-V. wrote the manuscript, and all others, especially C. M., performed and analysed the experiments and contributed to the discussion.

\section{References}

1. Hertog MG, Feskens EJ, Hollman PC, et al. (1993) Dietary antioxidant flavonoids and risk of coronary heart disease: the Zutphen Elderly Study. Lancet 342, 1007-1011.

2. Sampson L, Rimm E, Hollman PC, et al. (2002) Flavonol and flavone intakes in US health professionals. J Am Diet Assoc 102, 1414-1420.

3. Manach C, Scalbert A, Morand C, et al. (2004) Polyphenols: food sources and bioavailability. Am J Clin Nutr 79, $727-747$.

4. Perez-Vizcaino F, Bishop-Bailley D, Lodi F, et al. (2006) The flavonoid quercetin induces apoptosis and inhibits JNK activation in intimal vascular smooth muscle cells. Biochem Biophys Res Commun 346, 919-925.

5. Perez-Vizcaino F, Duarte J \& Andriantsitohaina R (2006) Endothelial function and cardiovascular disease: effects of quercetin and wine polyphenols. Free Radic Res $\mathbf{4 0}$, 1054-1065.

6. Perez-Vizcaino F, Duarte J, Jimenez R, et al. (2009) Antihypertensive effects of the flavonoid quercetin. Pharmacol Rep 61, 67-75.

7. Loke WM, Proudfoot JM, Hodgson JM, et al. (2010) Specific dietary polyphenols attenuate atherosclerosis in apolipoprotein E-knockout mice by alleviating inflammation and endothelial dysfunction. Arterioscler Thromb Vasc Biol 30, 749-757.

8. Duarte J, Perez Vizcaino F, Utrilla P, et al. (1993) Vasodilatory effects of flavonoids in rat aortic smooth muscle. Structureactivity relationships. Gen Pharmacol 24, 857-862.

9. Ibarra M, Perez-Vizcaino F, Cogolludo A, et al. (2002) Cardiovascular effects of isorhamnetin and quercetin in isolated rat and porcine vascular smooth muscle and isolated rat atria. Planta Med 68, 307-310.

10. Duarte J, Perez-Palencia R, Vargas F, et al. (2001) Antihypertensive effects of the flavonoid quercetin in spontaneously hypertensive rats. Br J Pharmacol 133, 117-124.

11. Ihm SH, Lee JO, Kim SJ, et al. (2009) Catechin prevents endothelial dysfunction in the prediabetic stage of OLETF rats by reducing vascular NADPH oxidase activity and expression. Atherosclerosis 206, 47-53.

12. Pignatelli P, Pulcinelli FM, Celestini A, et al. (2000) The flavonoids quercetin and catechin synergistically inhibit platelet function by antagonizing the intracellular production of hydrogen peroxide. Am J Clin Nutr 72, 1150-1155.

13. Pignatelli P, Di Santo S, Buchetti B, et al. (2006) Polyphenols enhance platelet nitric oxide by inhibiting protein kinase C-dependent NADPH oxidase activation: effect on platelet recruitment. FASEB J 20, 1082-1089. 
14. Silberberg M, Morand C, Manach C, et al. (2005) Coadministration of quercetin and catechin in rats alters their absorption but not their metabolism. Life Sci 77, 3156-3167.

15. Lopez-Lopez G, Moreno L, Cogolludo A, et al. (2004) Nitric oxide (NO) scavenging and NO protecting effects of quercetin and their biological significance in vascular smooth muscle. Mol Pharmacol 65, 851-859.

16. Jimenez R, Andriambeloson E, Duarte J, et al. (1999) Involvement of thromboxane $A_{2}$ in the endothelium-dependent contractions induced by myricetin in rat isolated aorta. $\mathrm{BrJ}$ Pharmacol 127, 1539-1544.

17. Cogolludo A, Moreno L, Frazziano G, et al. (2009) Activation of neutral sphingomyelinase is involved in acute hypoxic pulmonary vasoconstriction. Cardiovasc Res $\mathbf{8 2}$, 296-302.

18. Lodi F, Jimenez R, Moreno L, et al. (2008) Glucuronidated and sulfated metabolites of the flavonoid quercetin prevent endothelial dysfunction but lack direct vasorelaxant effects in rat aorta. Atherosclerosis 204, 34-39.

19. Perez-Vizcaino F, Cogolludo AL, Zaragoza-Arnaez F, et al. (1999) Vasodilator effects of sodium nitroprusside, levcromakalim and their combination in isolated rat aorta. Br J Pharmacol 128, 1419-1426.

20. Tallarida RJ (2001) Drug synergism: its detection and applications. J Pharmacol Exp Ther 298, 865-872.

21. Perez-Vizcaino F, Ibarra M, Cogolludo AL, et al. (2002) Endothelium-independent vasodilator effects of the flavonoid quercetin and its methylated metabolites in rat conductance and resistance arteries. J Pharmacol Exp Ther 302, 66-72.
22. Lam CS, Borlaug BA, Kane GC, et al. (2009) Age-associated increases in pulmonary artery systolic pressure in the general population. Circulation 119, 2663-2670.

23. Sanchez M, Lodi F, Vera R, et al. (2007) Quercetin and isorhamnetin prevent endothelial dysfunction, superoxide production, and overexpression of $\mathrm{p} 47 \mathrm{phox}$ induced by angiotensin II in rat aorta. J Nutr 137, 910-915.

24. Sanchez M, Galisteo M, Vera R, et al. (2006) Quercetin downregulates NADPH oxidase, increases eNOS activity and prevents endothelial dysfunction in spontaneously hypertensive rats. $J$ Hypertens $\mathbf{2 4}, 75-84$.

25. Halliwell B (2008) Are polyphenols antioxidants or prooxidants? What do we learn from cell culture and in vivo studies? Arch Biochem Biophys 476, 107-112.

26. Cogolludo A, Frazziano G, Briones AM, et al. (2007) The dietary flavonoid quercetin activates BKCa currents in coronary arteries via production of $\mathrm{H}_{2} \mathrm{O}_{2}$. Role in vasodilatation. Cardiovasc Res 73, 424-431.

27. Cirico TL \& Omaye ST (2006) Additive or synergetic effects of phenolic compounds on human low density lipoprotein oxidation. Food Chem Toxicol 44, 510-516.

28. Manach C, Williamson G, Morand C, et al. (2005) Bioavailability and bioefficacy of polyphenols in humans. I. Review of 97 bioavailability studies. Am J Clin Nutr 81, 230S-242S.

29. Lodi F, Jimenez R, Moreno L, et al. (2008) Glucuronidated and sulfated metabolites of the flavonoid quercetin prevent endothelial dysfunction but lack direct vasorelaxant effects in rat aorta. Atherosclerosis 204, 34-39.

30. Bieger J, Cermak R, Blank R, et al. (2008) Tissue distribution of quercetin in pigs after long-term dietary supplementation. J Nutr 138, 1417-1420. 